\title{
Filigrane
}

Écoutes psychanalytiques

\section{Les conflits suscités par la rencontre clinique avec l'altérité culturelle et leurs effets sur le contre-transfert}

\section{Nathalie Tissières et Irène Krymko-Bleton}

Volume 27, numéro 1, 2018

URI : https://id.erudit.org/iderudit/1055604ar

DOI : https://doi.org/10.7202/1055604ar

Aller au sommaire du numéro

Éditeur(s)

Revue Santé mentale au Québec

ISSN

1192-1412 (imprimé)

1911-4656 (numérique)

Découvrir la revue

Citer cet article

Tissières, N. \& Krymko-Bleton, I. (2018). Les conflits suscités par la rencontre clinique avec l'altérité culturelle et leurs effets sur le contre-transfert. Filigrane, 27(1), 81-97. https://doi.org/10.7202/1055604ar
Résumé de l'article

La rencontre avec "l'autre étranger " suscite souvent des réactions qui, en clinique, peuvent se manifester au travers de ce qu'on appelle le contre-transfert culturel. Le présent article a pour objectif d'identifier quelques formes de contre-transfert culturel fréquemment représentées chez les participants de cette recherche (sept psychologues d'approche psychodynamique) et porte spécifiquement sur ses formes les plus conflictuelles, voire potentiellement problématiques. Les participants ont été rencontrés à deux reprises. Lors de la première entrevue, il leur a été demandé de commenter des dessins d'enfants de différentes origines. Lors de la suivante, ils ont été questionnés sur leur pratique en situation interculturelle. L'analyse qualitative des données a mené à l'identification de trois formes de contre-transfert culturel : la réticence à considérer l'origine des patients, le malaise autour des inégalités ethno-raciales et un ressenti de colère ou le sentiment d'être heurté par certaines attitudes de patients appartenant à une minorité culturelle. Des hypothèses explicatives de ces résultats et une réflexion sur les conditions permettant de dépasser certaines formes de contre-transfert culturel plus problématiques sont proposées dans la discussion. 


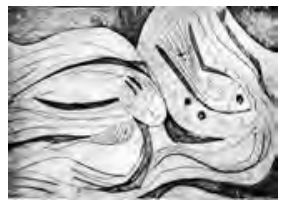

\title{
Les conflits suscités par la rencontre clinique avec l'altérité culturelle et leurs effets sur le contre-transfert
}

\author{
NathalieTissières \\ Irène Krymko-Bleton
}

\begin{abstract}
Résumé: La rencontre avec «l'autre étranger» suscite souvent des réactions qui, en clinique, peuvent se manifester au travers de ce qu'on appelle le contre-transfert culturel. Le présent article a pour objectif d'identifier quelques formes de contre-transfert culturel fréquemment représentées chez les participants de cette recherche (sept psychologues d'approche psychodynamique) et porte spécifiquement sur ses formes les plus conflictuelles, voire potentiellement problématiques. Les participants ont été rencontrés à deux reprises. Lors de la première entrevue, il leur a été demandé de commenter des dessins d'enfants de différentes origines. Lors de la suivante, ils ont été questionnés sur leur pratique en situation interculturelle. L'analyse qualitative des données a mené à l'identification de trois formes de contre-transfert culturel: la réticence à considérer l'origine des patients, le malaise autour des inégalités ethno-raciales et un ressenti de colère ou le sentiment d'être heurté par certaines attitudes de patients appartenant à une minorité culturelle. Des hypothèses explicatives de ces résultats et une réflexion sur les conditions permettant de dépasser certaines formes de contre-transfert culturel plus problématiques sont proposées dans la discussion.
\end{abstract}

Mots clés: contre-transfert culturel; psychothérapie interculturelle; approche psychodynamique; dessins d'enfants; racisme.

\begin{abstract}
The clinical encounter with "the other stranger" often gives rise to reactions that can take the form of cultural countertransference. The purpose of this paper is to identify a few forms of cultural countertransference that were frequently represented among the participants of this research (seven psychologists of the psychodynamic approach). Emphasis is then placed on the most conflicted or potentially problematic forms of cultural countertransference. Participants were met twice. During the first interview, they were asked to comment on drawings of children from different cultural backgrounds. In the second interview, participants answered questions about their practice in the intercultural context. The qualitative analysis of the data highlighted three forms of cultural countertransference: the reluctance to consider the cultural origin of the patients, the discomfort around ethno-racial inequalities and feelings of anger or of being stricken by
\end{abstract}


certain attitudes of patients of cultural minorities. Explanatory hypotheses of the results are discussed and, after careful consideration, conditions for overcoming problematic forms of cultural countertransference are proposed in the discussion. Key Words: cultural countertransference; intercultural psychotherapy; psychodynamic approach; children's drawings; racism.

et article, écrit dans le cadre d'une thèse de doctorat en psychologie, s'appuie sur des entrevues de recherche où il a été demandé à des psychologues d'origines diverses pratiquant au Québec de commenter des dessins d'enfants de différentes origines et de parler de leur conception de la clinique interculturelle. L'analyse des données a mené à un premier article qui aborde les enjeux spécifiques aux psychologues immigrants, tels que le rôle de leur identité culturelle dans leurs interprétations de dessins d'enfants, leur relation avec leurs patients immigrants et l'impact d'expériences de discrimination sur leur identité professionnelle (Tissières et KrymkoBleton, 2018). Ce deuxième article porte quant à lui sur les expériences de l'ensemble des psychologues interrogés (immigrants et québécois ${ }^{1}$ ) et se concentre spécifiquement sur la question du contre-transfert culturel.

Le contre-transfert culturel se définit selon Baubet et Moro (2009) comme la manière dont le thérapeute se positionne intérieurement par rapport à l'altérité du patient. Autrement dit, il s'agit des réactions explicites et implicites, conscientes et inconscientes du clinicien aux affiliations de son patient, en situation clinique (Baubet et Moro, 2009). Ces réactions peuvent être suscitées par la culture au sens strict (par exemple la langue ou les manières de faire ou de voir le monde), mais aussi par des concepts connexes tels que l'immigration, la couleur de peau, les traumatismes de guerre ou encore la discrimination vécue par les patients. Certains psychologues interrogés ont parlé directement de quelques-unes de leurs réactions, mais il a surtout été possible de supposer la nature de leur contre-transfert culturel indirectement, en le déduisant à partir des façons de dire.

Le contre-transfert culturel peut être un guide précieux pour la compréhension du patient comme il peut conduire à des écueils thérapeutiques s'il n'est pas élaboré (Rouchon et al., 2009). Même s'il est bien sûr unique à chaque relation thérapeutique, il y aurait des formes de contre-transfert culturel plus fréquentes que d'autres: le déni de la différence, sa réification, la fascination de l'exotique ou des affects de culpabilité, pitié et agressivité (Comas-Díaz et Jacobsen, 1991; Rouchon, 2007). Dans la littérature, ce sont la plupart du temps les formes de contre-transfert culturel potentiellement 
problématiques (ou du moins conflictuelles) qui sont relevées et c'est également le parti pris de cet article. J' ${ }^{2}{ }^{2}$ donc laissé de côté les extraits d'entrevues (majoritaires chez certains participants, plus rares chez d'autres) laissant supposer un certain dégagement, une aisance dans la rencontre avec «l'autre étranger» (Rouchon et al., 2009).

Les conflits suscités par la différence culturelle ne sont pas problématiques en soi. Comme l'avance Rousseau (2008), «dans les sociétés métissées, le malaise de la rencontre avec l'autre fait partie d'un enrichissement social». Toutefois, en clinique, ces conflits peuvent devenir des obstacles au bon déroulement de la thérapie quand ils sont évités, agis, ou non élaborés. L'objectif de cet article est donc de répondre à la question suivante: quelles sont les formes de contre-transfert culturel potentiellement problématiques les plus représentées parmi les participants de cette recherche? J'ai cherché à répondre à cette question en m'adressant à des psychologues d'orientation psychodynamique pour plusieurs raisons: l'intérêt pour le contre-transfert propre à cette approche, ma familiarité avec elle et le désir d'une certaine uniformité dans mon échantillon.

\section{Méthodologie}

Pour participer à la recherche, les psychologues devaient travailler en clinique infantile, se réclamer de l'approche psychodynamique et avoir déjà rencontré des patients provenant de minorités culturelles. Quatre participants sont immigrants de première ou deuxième génération, trois sont québécois depuis plusieurs générations.

Ces psychologues ont été rencontrés à deux reprises. Lors de la première entrevue, des dessins d'enfants de trois origines différentes (Haïti, Québec et Amérique latine), sélectionnés principalement en fonction de leur susceptibilité de susciter des interprétations liées aux origines, leur ont été présentés. Les dessins avaient été recueillis dans le cadre d'une autre recherche ${ }^{3}$ où il avait été demandé à des enfants d'écoles primaires de Montréal de produire quatre dessins: un dessin libre, un bonhomme «le plus beau possible», leur famille réelle et leur famille rêvée. Après avoir informé les participants du sexe, de l'âge et de l'origine des enfants, je les ai invités à me parler de ce que ces dessins leur évoquaient. Il s'agissait donc d'une entrevue non structurée où mes interventions se limitaient à soutenir l'élaboration. Cette première rencontre a servi de déclencheur aux réflexions recueillies dans l'entrevue semi-structurée suivante, qui abordait leur pratique clinique, ainsi que leur histoire d'immigration dans le cas des psychologues immigrants. 
Les analyses se sont déroulées en plusieurs étapes itératives: imprégnation dans les données, analyse thématique (Paillé et Mucchielli, 2003) et analyses minutieuses selon une méthode développée par Krymko-Bleton (2014 a, 2014 b, 2016). Cette méthode consiste entre autres à porter une attention particulière au contexte relationnel de la rencontre, notamment au moyen de l'analyse des places interlocutives (Flahault, 1978). Elle implique aussi de suivre les fils associatifs, soit la suite des idées ou les ruptures du discours révélant éventuellement les préoccupations du sujet, conscientes et inconscientes. Mon attention s'est également portée sur tout ce qui témoigne, dans les façons de dire, de la conflictualité du sujet: les confusions, les contradictions, les fautes, les hésitations, les rires, etc. Les résultats prennent la forme d'interprétations, toujours hypothétiques, mais appuyées par des extraits de verbatim. Précisons aussi qu'il n'est pas toujours possible de déterminer la part de l'inconscient dans les réactions contre-transférentielles analysées. Souvent, l'analyse des façons de dire permet de détecter un conflit que l'on peut supposer en partie conscient chez le participant, même s'il n'est pas exprimé explicitement. De façon générale, la dimension strictement inconsciente du contre-transfert n'a pas pu être abordée dans cette recherche, le nombre restreint d'entrevues ne permettant pas d'accéder à un tel matériel.

\section{Résultats}

Je tiens à préciser que les formes de contre-transfert culturel décrites ici sont à comprendre comme des tendances générales, puisque toute réaction contre-transférentielle est par définition une expérience unique et singulière donc nullement reproductible et standardisable (Rouchon, 2007). Le but est de poser certains repères, par une catégorisation inévitablement discutable, mais utile à la réflexion. Les trois formes de contre-transferts culturels retenues sont les suivantes: la réticence à considérer l'origine des patients, le malaise face aux inégalités ethno-raciales (une sous-catégorie du point précédent) et un ressenti de colère ou le sentiment d'être heurté par certaines attitudes de patients appartenant à une minorité culturelle.

\section{Réticence à considérer l'origine des patients}

Plus de la moitié des psychologues rencontrés (4 sur 7) ont montré une certaine réticence à prendre en compte l'origine des enfants dans leurs interprétations des dessins. Lorsqu'ils font des interprétations culturelles, ils ponctuent leur discours de termes illustrant leur besoin de se montrer extrêmement prudents. C'est le cas dans le commentaire suivant à propos 
de la présence d'une nièce dans le dessin de famille d'une fillette d'origine haïtienne (Figure 1):

Je sais pas si le fait d'avoir une nièce dans sa famille tsé y a déjà beaucoup d'enfants hein, est-ce que, pourquoi elle inclut la nièce, c'est comme si quelque part les, la famille nucléaire c'est, mais ça c'est un préjugé que j’ai, mais c'est que les réseaux familiales [sic] dans les familles haïtiennes sont j'ai l'impression beaucoup plus importants que dans nos familles nord-américaines, je pense en tous cas donc euh, peut-être que c'est la trace de ça [...]

L'analyse des façons de parler de cet extrait laisse supposer que cette participante n'ose pas complètement associer culture haïtienne et familles élargies. Les signes d'hésitation s'accumulent: «je ne sais pas", "comme si quelque part», «j'ai l'impression», «je pense en tous cas», pour ne citer que les plus manifestes. En mentionnant que ce qu’elle avance est «un préjugé», elle dévoile sa crainte de généraliser abusivement, voire de discriminer, comme si nommer une différence (une conception différente de la famille) revenait à le faire. L'enfant étant absente, on peut supposer que son discours

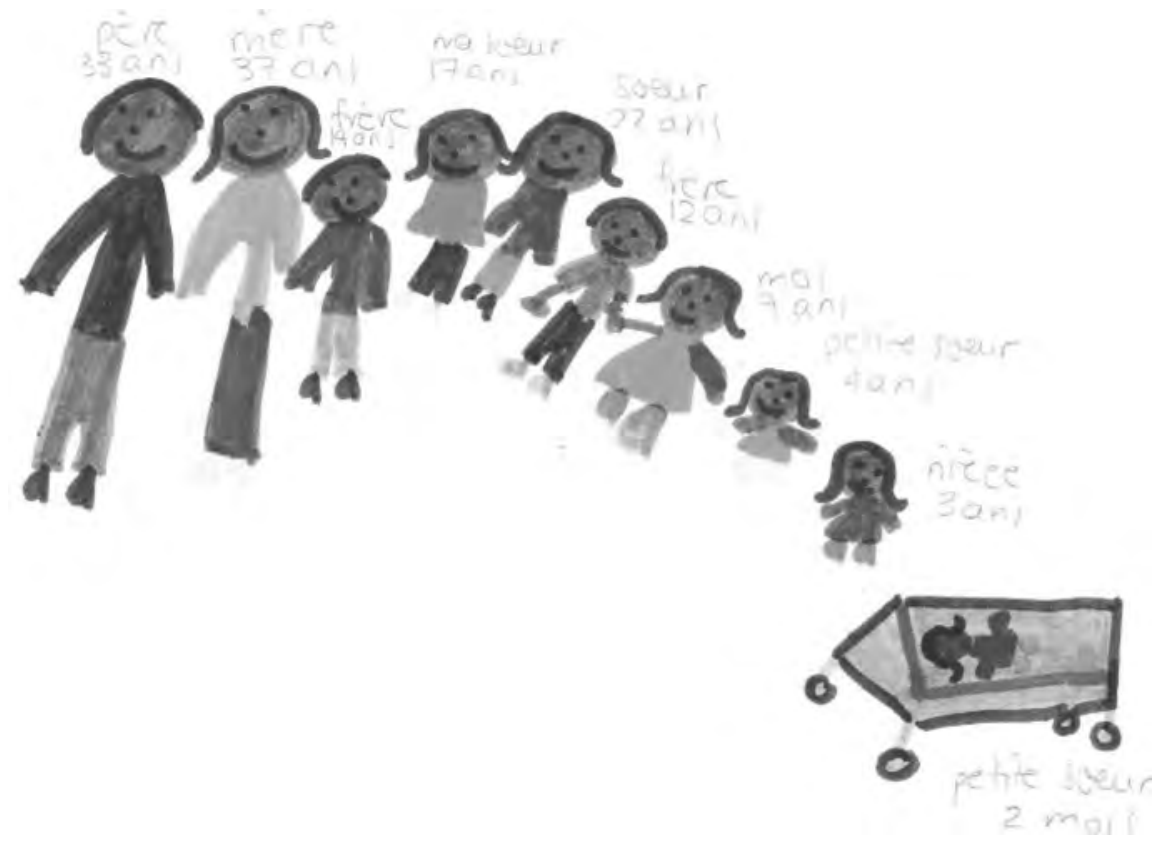

Figure 1. La «famille réelle» d'une enfant d'origine haïtienne 
est contraint avant tout par mon regard sur elle (ou le regard des autres personnes qui pourraient avoir accès à ses propos dans son imaginaire, en dépit de la confidentialité assurée).

Malgré leur réticence initiale, la plupart des participants ont, à petites doses, tenu compte de la culture dans leurs interprétations. Pourtant, quand on leur demande quels ont été leurs points de repère pour leurs interprétations, ils ne pensent pas à nommer celle-ci, une participante allant jusqu'à nier son influence même si certaines de ses interprétations y faisaient explicitement référence. De façon générale se dégage l'impression que les participants pensent rapidement à la culture ou aux notions connexes (c'est «l'éléphant dans la pièce»), mais que quelque chose les empêche de suivre pleinement leur idée première.

La réticence à considérer l'origine des patients se traduit aussi par une certaine banalisation de l'influence de la culture en clinique, comme le témoigne le peu d'intérêt de la grande majorité des participants pour les formations sur le sujet. Une participante avance par exemple qu' «il y a pas de frontières de cultures », affirmation qui semble faire fi des enjeux inévitablement suscités par la confrontation à l'altérité culturelle.

Une autre participante se montre plus ambivalente. Son propos pourrait se résumer ainsi: «la culture du patient, c'est important, mais pas plus que tout le reste». Elle affirme ainsi d'abord tenir compte de la culture, notamment des différences entre elle et ses patients qui pourraient causer des incompréhensions, puis nuance en disant que la différence est toujours présente, peu importe la culture puisque «chaque client arrive avec ses référents» et qu' «il y a donc toujours une part d'inconnu à apprivoiser». Son ambivalence et sa conflictualité transparaissent aussi dans l'extrait suivant (les hésitations, les termes "peut-être» versus «souvent», «mais», et l'exemple choisi témoignant d'une association d'idées particulière entre l'origine culturelle et l'hospitalisation):

Je vais peut-être plus poser de questions sur la culture ou sur euh, sur l'immigration souvent, mais comme je vais poser la question sur un enfant qui a été hospitalisé euh $[\ldots]$

Un psychologue immigrant répète quant à lui à sept reprises qu’il est très «facile» de travailler avec des patients québécois. Il ne rencontrerait pas de barrières culturelles, ce qui me surprend, car il y a eu entre nous de légers, mais évidents malentendus culturels ou linguistiques. Chez lui, ce possible 
déni des difficultés peut probablement être expliqué en grande partie par son besoin d'être reconnu dans ses compétences, ce qui n’a pas toujours été le cas en raison de sa position fragile d'immigrant (Tissières et KrymkoBleton, 2018).

Concluons cette partie par les mots éloquents d'une participante qui se montre lucide à propos du phénomène de la banalisation de la culture en clinique:

[La culture] est plutôt euh... je ne sais pas, scotomisée, c'est plutôt clivé dans le discours clinique c'est comme si ça n'existait pas. [...] On ne l'abordera pas nécessairement facilement avec les gens, on le sait, on en tient compte, on y pense nous dans notre clinique, mais c'est pas si facile à arriver pis parler des éléments culturels qui peuvent affecter le... ajouter une couleur particulière au problème, tsé c'est ça, non je pense qu'on n'en tient pas assez compte en fait, pis on est gêné d'aborder ça avec les personnes, les parents.

\section{Malaise autour des inégalités ethno-raciales}

Le dessin du "plus beau bonhomme possible» de la fillette originaire d'Haïti (Figure 2) aurait pu susciter des commentaires sur les conséquences des inégalités ethno-raciales sur l'image de soi d'une enfant. Cette dernière a choisi de dessiner un personnage blanc et blond alors que dans ses dessins de famille (réelle et rêvée), ses personnages ont la peau noire (Figure 1). Parmi les nombreuses explications possibles à cette particularité, une aurait pu être considérée: se pourrait-il que cette enfant ait associé la couleur de peau blanche à la beauté, car elle baigne dans une société où être noir est encore un désavantage à plusieurs niveaux (réussite scolaire, emploi, revenu)? Étonnamment, la grande majorité des psychologues ( 6 sur 7 ) n’ont pas émis d'hypothèses explicites en ce sens. Les mots «racisme», "discrimination», «privilège blanc» ou «inégalité» sont absents de leur discours. Pourtant, plusieurs semblent en fait avoir pensé à cette hypothèse, mais se sont ensuite empressés de se diriger vers d'autres interprétations.

Une psychologue affirme par exemple à propos du bonhomme blanc de la fillette haïtienne «ne pas savoir ce que ça veut dire [...] par rapport à ses propres origines, si c'est important ou pas", puis évoque que ce n'est que très récemment que les enfants noirs dessinent des bonshommes de leur propre couleur de peau. Pourquoi ressent-elle le besoin de faire ce commentaire? On pourrait supposer qu'il a pour fonction de banaliser cette particularité 


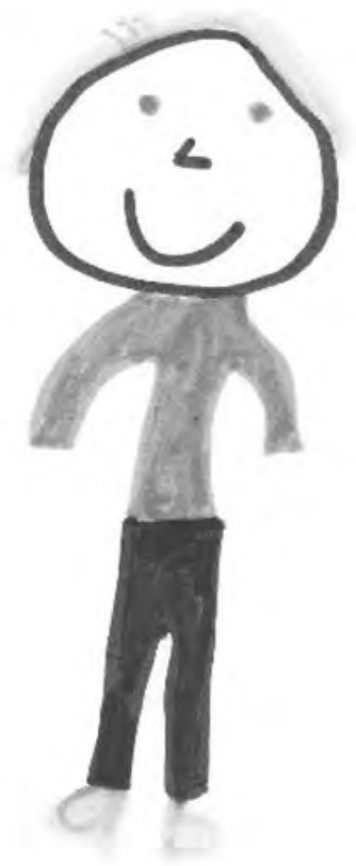

Figure 2. Le «plus beau bonhomme possible» d'une enfant d'origine haïtienne

(qu'une enfant noire dessine un bonhomme blanc était la norme dans le passé). Soulignons aussi la nature paradoxale de ce propos qui, tout en banalisant un phénomène, évoque implicitement un passé d'oppression. Pourtant, elle se dira ensuite "frappée qu'il ne soit pas brun», expression forte émise sur un ton qui dément le caractère anodin de ce choix de couleur de peau.

Un autre participant imagine d'abord un «idéal en conflit avec ce qu'elle est », mais ses associations l'écartent ensuite de cette première idée. Ce dessin lui rappelle un jeune patient québécois qui s’était dessiné en noir, car il aurait voulu être bronzé, comme le reste de sa famille qui était partie à la mer sans lui. La situation est donc dans ce cas renversée, puisqu'être noir (ou bronzé) est souhaité par un enfant. Ce clinicien a-t-il (inconsciemment peut-être) cherché à retourner dans une zone confortable, évitant ainsi de se questionner sur les raisons qui pourraient pousser une enfant haïtienne à désirer être blanche? Notons que l'idée d'exclusion, qui apparaît dans son 
exemple, laisse supposer que la question de la discrimination des personnes racisées ne lui a pas échappé complètement. Il avance d'ailleurs ensuite que ce serait selon lui «plus au niveau socio... de la sociologie qu'on trouverait peut-être des hypothèses ", comme s'il ne pouvait concevoir d'avoir luimême un double discours, à la fois psychodynamique et sociologique.

Une autre psychologue se questionne sur «l'identité de l'enfant» et sur sa façon de considérer "sa nouvelle culture». Elle semble penser qu'il y a des enjeux liés à la couleur de peau puisqu'elle note que le bonhomme «fait très idéal caucasien ». Mais le commentaire suivant suggère aussi un évitement des inégalités ethno-raciales:

Dans certains pays, on va beaucoup rechercher la rareté donc, quelque chose de beau c'est quelque chose qui est rare, qu'on voit pas beaucoup. Alors si tout le monde est brun, ce qui est beau, c'est les cheveux blonds.

Étrange hypothèse qu'on peut difficilement imaginer s'appliquer à cette petite Haïtienne qui vit dans un pays majoritairement blanc (la «rareté» est plutôt d'être noir). Elle lui permet de substituer la question de la couleur de peau (et de la discrimination qu'elle peut impliquer) par un sujet plus universel, le fait de désirer la rareté. Par ailleurs, sa formulation («dans certains pays», «on») est floue et très générale, ce qui pourrait être interprété comme une défense l'éloignant de la situation présente, celle d'une enfant d'origine haïtienne vivant au Québec. Par la suite, elle tente de tirer un parallèle avec la valorisation du surpoids dans les pays souffrant d'insécurité alimentaire. Cet argument censé appuyer sa thèse du désir de ce qui est rare est incohérent, puisque cette valorisation s'explique surtout par la richesse économique dont témoigne le surpoids. On pourrait donc présumer que cette psychologue a bel et bien associé la peau blanche à des privilèges, mais qu'elle a ressenti l'impératif de nier ce qui pourtant s'imposait à elle (dénégation).

Une autre psychologue fixe d'abord son attention sur des éléments du dessin qui ne concernent pas la couleur de peau (l'absence de robe chez une fille, le peu de couleurs des habits). Le thème de la couleur des habits laisse croire que la question de la couleur de peau affleure, mais qu'elle est mise à distance par déplacement. C'est uniquement à la fin de l'entrevue qu'elle aborde ce sujet, comme si quelque chose l'avait retenue avant. Elle supposera en effet un «rejet aussi de l'environnement tsé par rapport à son... à sa... le fait qu'elle a la peau noire», montrant ainsi qu'elle est consciente de 
l'existence de la discrimination. Son hésitation («à son... à sa...») dénote un malaise à parler de couleur de peau (terme tout de même plus acceptable que celui de «race»). Notons aussi que le terme d' «environnement» reste vague, moins menaçant que celui, par exemple, de société d'accueil.

\section{Ressenti de colère ou sentiment d'être heurté par certaines attitudes de patients appartenant à une minorité culturelle}

Même si beaucoup de participants ont eu généralement tendance à banaliser les difficultés possibles des rencontres interculturelles, certains d'entre eux ont aussi démontré, à certains moments, une capacité à nommer un contre-transfert culturel teinté d'affects tels que la colère ou le sentiment d'être heurté. Bien sûr, de tels affects peuvent survenir dans tout processus thérapeutique, peu importe l'origine ethnique. Mais il semblerait qu'ils soient particulièrement fréquents ou difficiles à dépasser lorsqu'ils sont suscités par la différence culturelle. Cette forme de contre-transfert n'est pas apparue dans les commentaires de dessins, mais lorsque les psychologues abordaient leur pratique. Cela s'explique certainement par la nature même de ce matériel, moins susceptible d'éveiller des affects intenses que des rencontres cliniques.

Voici un premier extrait qui illustre bien le ressenti de colère ou le sentiment d'être heurté d'une participante confrontée à des familles haïtiennes qui interrompent le suivi afin d'envoyer l'enfant se faire soigner en Haïti selon les croyances traditionnelles:

On nous annonce que le petit est parti dans la famille élargie en Haïti pis on sait qu'il va y avoir quelque chose de l'ordre de, je ne sais pas moi euh... j'ai oublié le terme de leur croyance païenne là [...] Là je ne comprends plus, je ne comprends pas tellement là les familles haïtiennes, ces aspectslà, ça... tsé je respecte, pis je me dis... pis ça me fâche, souvent ça me fâche je me dis ah, mais ça défait tout mon suivi ou tout ce que j'essaie de construire avec cet enfant-là, on dirait qu'ils ont pas confiance.

Cet extrait révèle (notamment par la répétition des verbes «comprendre» et «se fâcher» et le ton chargé d'affects) le désarroi de cette psychologue. Sa façon de parler du vaudou (la «croyance païenne» dont elle avait oublié le nom) laisse croire qu'elle conçoit les visions occidentales et traditionnelles de l'étiologie des maladies comme opposées et incompatibles. Derrière la colère et l'incompréhension se dévoile aussi une blessure 
provoquée par son impression qu'on ne lui fait pas confiance et qu'on ne reconnaît pas son suivi à sa juste valeur.

Un autre psychologue se dit «heurté» par un père haïtien dont le fils manquait souvent les rendez-vous et qui lui aurait, de façon évidente, menti sur les motifs d'absence. Il suppose que la relation de ce père à l'autorité qu'il représentait est en lien avec le régime politique qu'il a pu subir en Haïti, mais cette possible explication ne le satisfait pas. Il semble avoir manqué de clés (possiblement culturelles, politiques, historiques) pour parvenir à «se décentrer culturellement» (Baubet et Moro, 2009) afin de comprendre ce comportement et ainsi l'appréhender avec plus d'empathie.

Une troisième psychologue relate sa réaction lorsqu'une patiente d'origine arabe a commencé à porter le voile au cours du suivi. Elle aurait réalisé qu'elle trouvait cette décision "triste», même si "en élaborant avec elle, $\mathrm{y}$ avait quelque chose pour elle de très signifiant et de très positif». Cette patiente aurait mis fin au suivi, notamment pour des raisons financières. Toutefois, la psychologue semble s'interroger sur l'impact de son malaise par rapport au voile:

Fait que là elle a eu une possibilité [au public], mais je me demande si ça n'a pas contribué euh, je me demande. Le fait que j'avais un malaise par rapport au voile. Mais en même temps, ça nous a obligés à en parler ou elle a dû élaborer qu'est-ce qu'elle en pensait, comment elle voyait ça pis euh...

Cet extrait pourrait laisser entrevoir que cette clinicienne se défend contre l'idée que son malaise ait joué un rôle dans le bon déroulement de la thérapie. Elle semble essayer de se convaincre du contraire ( «mais en même temps»), soulignant les conséquences positives de son malaise. Les défenses transparaissent aussi dans les hésitations ( «pis euh») ou dans sa première phrase restée inachevée («contribué» - à quoi?).

Dans ces trois exemples, le contre-transfert est nommé, en partie élaboré, mais plus difficilement dépassé ou reconnu dans ses conséquences sur la thérapie.

\section{Discussion}

Cette recherche a permis d'identifier trois formes de contre-transfert culturel fréquentes et potentiellement problématiques parmi les participants de cette étude: la réticence à considérer l'origine des patients, le malaise autour des inégalités ethno-raciales et un ressenti de colère ou le 
sentiment d'être heurté par certaines attitudes de patients appartenant à une minorité culturelle.

Les deux premières formes de contre-transfert relevées dévoilent une tendance à l'évitement par rapport aux questions culturelles, en particulier quand elles touchent aux inégalités ethno-raciales. On pourrait aussi parler de censure, car, de manière générale, la culture (et les notions connexes telles que l'immigration, la discrimination, etc.) était bien présente dans l'esprit des psychologues, mais quelque chose les empêchait de s'aventurer plus en avant sur cette voie. Un des résultats importants de cette recherche est le constat que les conflits inévitablement suscités par la rencontre avec l'autre étranger se sont présentés de façon privilégiée sur le mode de l'évitement. Cela est vrai dans ce contexte bien spécifique de recherche, mais il est également possible, voire probable, qu'ils puissent aussi se retrouver fréquemment au sein de la clinique. Comment expliquer cette tendance à l'évitement?

Une première explication possible est d'ordre sociologique et concerne le poids du «politiquement correct» aujourd'hui (Neville et al., 2013). Il y aurait un climat où le seul fait de nommer une différence pourrait être considéré comme raciste (Fassin, 2009). Il serait de bon ton d'être «aveugle culturellement », soit de prétendre que la culture ou la couleur de peau n'ont aucun impact sur nos vies (Neville et al., 2013). Il se pourrait donc que les psychologues soient influencés par ce climat qui prévaut dans les sociétés occidentales, même si bien sûr il peut prendre différentes formes selon les régions.

Une autre hypothèse, plus psychologique, est avancée par Cooper (2010). Le simple fait de mentionner une différence raciale dans un contexte groupal soulèverait souvent une anxiété intense chez les membres blancs du groupe, même si cette différence est complètement visible et évidente pour tous. Il est vrai qu'il peut parfois être difficile de «nommer des caractéristiques phénotypiques sans être pris dans le mouvement de reproduire et d'imposer cette catégorisation» (Delanoë et Moro, 2016, p. 207). Mais ce phénomène semble avoir une ampleur qui va au-delà de cette considération. Les personnes blanches en Amérique du Nord vivraient dans un environnement social qui les exposerait peu à la réalité des minorités culturelles (existence de quartiers majoritairement blancs et sous-représentation des minorités culturelles dans les médias par exemple). Elles seraient donc protégées et isolées du stress racial, ce qui réduirait leur capacité à le tolérer (DiAngelo, 2011). Une forte anxiété apparaitrait donc facilement 
et susciterait des mouvements défensifs, tels que l'affichage d'émotions comme la colère, la peur, la culpabilité, et des comportements comme l'argumentation, le silence ou la fuite. L'anxiété provoquée par le simple fait de nommer une différence culturelle pourrait aussi provenir de la projection d'un «inconscient raciste» (Davids, 2003), qui serait présent en chacun de nous, sur la personne qui a nommé explicitement une différence culturelle (celle-ci étant accusée de racisme) (Cooper, 2010). De façon plus générale, l'angoisse suscitée par la différence culturelle peut être comprise au travers du concept d'«inquiétante étrangeté» (Freud, 1919). Ce concept freudien, repris par Kristeva (1988), fait référence à des angoisses infantiles refoulées qui se montrent à nouveau (Freud, 1919). L'intime surgit alors comme étranger, inconnu, au point d'en être effrayant (Menès, 2004). Ce phénomène surviendrait par exemple lors de situations déconcertantes (Freud, 1919). Le contact parfois décontenançant avec l'autre étranger pourrait donc être vu comme une situation particulièrement propice à l'apparition d'un sentiment d'inquiétante étrangeté. Par ailleurs, d'autres explications d'ordre psychanalytique ont été avancées pour comprendre la fréquence de réactions fortes et même hostiles face à l'étranger. Pour Kaës (1998), la différence culturelle représenterait la "troisième différence», celle qui se prêterait bien à la métaphorisation des deux premières différences, soit la différence entre humain et non-humain (première différence) et la différence des sexes et des générations (deuxième différence). La différence culturelle serait ainsi infiltrée par les angoisses des deux premières. L'étranger nous ramènerait donc à des questions psychologiques fondamentales comme celle de l'identité, de l'identité sexuelle et du statut de sujet (Fermi, 1998).

En outre, la prépondérance d'une tendance à l'évitement des questions culturelles pourrait aussi s'expliquer par des motifs propres à l'approche psychodynamique. Mentionnons d'abord la crainte des étiquettes et des diagnostics qui risqueraient d'enfermer une personne dans une pathologie ou dans un attribut culturel.

Par ailleurs, l'intérêt accru des psychanalystes pour la réalité psychique les pousserait parfois à vouloir s'extraire des questions raciales (Cooper, 2010). Mestre et Moro avancent que le politique (qui comprend les inégalités ethno-raciales) rendrait aussi compte des limites de la psychanalyse. Selon ces auteures, il existerait une «résistance de la psychanalyse au politique» (Mestre et Moro, 2012, p. 269) qui pourrait provenir du fait que certains psychologues peinent à accepter que leurs théories seules ne suffisent pas pour comprendre la souffrance d'un patient. Pour Moro (2002), 
les cliniciens rechigneraient à tenir un discours sur la différence, car ils sont conscients qu'ils le font inévitablement depuis une certaine "place». Il «n'existe pas une approche neutre du racisme [...]. C'est donc (selon la dialectique hégélienne) d'une place de maître ou d'esclave qu'on tient un discours sur la différence» (Moro, 2002, p. 346). Ce discours aurait donc inévitablement des implications sociales, voire politiques et morales, qui peuvent être perçues comme contraires à l'éthique clinique.

En outre, Cooper (2010) avance que les psychologues d'approche psychodynamique seraient hypervigilants face aux dangers du passage à l'acte et confondraient facilement l'agir clinique avec toute forme d'action. En clinique, cela pourrait se traduire par la crainte de soulever un sujet sans qu'il soit amené explicitement par un patient, surtout s'il est aussi délicat que celui de la culture ou des inégalités ethno-raciales.

Pour toutes ces raisons, le rapport à la culture de certains psychologues d'orientation psychodynamique peut être conflictuel (Cooper, 2010). Toutefois, il est important de souligner que cette approche comporte aussi certains a priori théoriques qui la rendent particulièrement apte à penser la culture dans toute sa complexité, dont l'importance donnée au contre-transfert.

Vient maintenant la question de l'impact de ces deux formes de contretransfert culturel (la réticence à considérer l'origine du patient et le malaise face aux inégalités ethno-raciales) dans la clinique. Sont-elles problématiques? De façon générale, il semblerait que les patients se sentent incompris, vivent de la frustration, sont moins enclins à se dévoiler et jugent moins crédibles les psychologues qui évitent le sujet de la race, de la culture ou des inégalités ethno-raciales (Thompson et Jenal, 1994). Par ailleurs, ne pas reconnaître la réalité de la discrimination pourrait favoriser un sentiment de culpabilité chez les patients, les laissant penser qu'ils sont les uniques responsables de la violence qu'ils subissent (Mestre et Moro, 2012). Pour certains auteurs, toute attitude d'aveuglement culturel devrait être considérée comme raciste, non dans son intention, mais par ses effets (Ciclitira et Foster, 2012). Toutefois, certaines nuances méritent d'être apportées. Est-il judicieux de mettre sur le même plan un racisme exprimé ouvertement et un «racisme» involontaire, inconscient, fruit d'un manque de connaissances, du peu de sensibilisation aux enjeux de rapports de pouvoir ou d'une difficulté à percevoir les conflits psychiques qui inévitablement surviennent en présence de l'altérité culturelle? Il me semble, pour ma part, qu'il est crucial de reconnaître les effets délétères (erreurs diagnostiques, risque d'un nouvel 
assujettissement, jugements arbitraires sur un patient) de comportements tels que l'évitement des questions culturelles. Cependant, j'hésite à parler de racisme dans ces cas-là, tant ce terme est associé à des comportements qui n'ont rien en commun avec ceux des psychologues interrogés. Il s'agirait d'inventer un nouveau mot. Faute de mieux, peut-être pourrions-nous parler d'un «contre-transfert culturel aux conséquences potentiellement racistes»?

Soulignons aussi qu'il n'est pas toujours simple de tracer une ligne entre évitement défensif et saine prudence. Il n'est évidemment pas souhaitable d'aborder directement des questions culturelles en clinique sans égard au rythme et à la sensibilité du patient. Certaines des raisons évoquées ci-dessus pour comprendre la tendance à l'évitement de ces sujets peuvent, dans certains cas, faire pencher la balance vers une certaine pudeur, une retenue qui, loin de nuire au patient, le protège. Toutefois, les résultats de cette recherche indiquent que cet évitement a une ampleur qui ne peut être attribuée uniquement à une saine prudence.

La troisième et dernière forme de contre-transfert culturel décrite, soit un ressenti de colère ou le sentiment d'être heurté par certaines attitudes de patients appartenant à une minorité culturelle soulève la question suivante: comment, dans ces conditions, parvenir à poursuivre un travail thérapeutique?

De façon générale, on considère que repérer et analyser les mouvements contre-transférentiels affectifs et culturels du thérapeute permettent d'éviter l'arbitraire, l'ajout de la violence à la violence ou tout autre agir malheureux (Huqueleux, 2016). Mestre et Moro parlent d'une «vigilance critique par l'analyse du contre-transfert culturel, c'est-à-dire l'analyse des idéologies et des rapports de pouvoir en jeu qui peuvent déformer l'appréciation clinique» (Mestre et Moro, 2012, p. 270). Aux prises avec un contre-transfert culturel qui lui a permis de reconnaître ses propres fantasmes racistes inconscients, Huqueleux rapporte que pour poursuivre un travail de qualité, il lui a été nécessaire de «ramener du tiers» (Huqueleux, 2016, p. 188) et ainsi avoir accès à des regards extérieurs pour penser les mouvements transféro-contre-transférentiels en jeu. Précisons que ce travail d'équipe a le potentiel d'être beaucoup plus riche et nuancé si les différents professionnels consultés sont diversifiés au niveau de leurs origines (Cooper, 2010). Malheureusement, les psychologues en pratique privée ne bénéficient pas d'un tel cadre. Il se pourrait d'ailleurs que ce soit ce qui a manqué aux participants qui ont témoigné de difficultés à dépasser 
un contre-transfert culturel chargé d'affects pénibles. Dans certains cas, la théorie peut faire office de tiers, mais les psychologues interrogés, pour la plupart non formés aux thérapies interculturelles, n'ont pas non plus eu accès (ou cherché à avoir accès) à ce support.

Terminons en soulignant l'existence d'un cercle vicieux. Plusieurs des psychologues interrogés s'empêchent de donner une grande importance aux questions culturelles pour les raisons évoquées ci-dessus. Ils ne sont donc pas non plus portés à se former sur ce sujet. Mais lorsque la culture devient saillante et suscite chez eux un contre-transfert affectif intense, ils sont alors peu outillés pour y faire face et ressentent, on peut l'imaginer, une certaine gêne à demander de l'aide. L'ouverture à des approches "transculturelles» sans craindre de perdre l'essence de leur approche clinique représente un défi pour plusieurs des participants et, je le suppose, pour de nombreux psychologues.

Nathalie Tissières

nathalie.tissieres@gmail.com

Irène Krymko-Bleton

krymko-bleton.irene@uqam.ca

\section{Notes}

1. Pour éviter des formulations lourdes, j'utilise le terme «québécois» pour parler d'une personne qui vit au Québec depuis plusieurs générations. J'oppose ici ce terme à celui d' "immigrant», même si en réalité les immigrants ont souvent des identités fluides ou multiples et peuvent se considérer ou être considérés comme québécois.

2. Cet article a été écrit en étroite collaboration avec ma directrice de thèse, Irène KrymkoBleton, mais en raison de la nature personnelle de certains propos, la première personne du singulier a été privilégiée.

3. CoPsyEnfant, la construction de l'identité aujourd'hui: construction psychique et psychopathologie de l'enfant dans les nouveaux liens familiaux et sociaux.

\section{Références}

Baubet, T. et Moro, M. R. (2009). Psychopathologie transculturelle. Paris: Masson.

Comas-Díaz, L., et Jacobsen, F. M. (1991). Ethnocultural transference and countertransference in the therapeutic dyad. American Journal of Orthopsychiatry, 61 (3), 392-402.

Cooper, A. (2010). Institutional Racism: Can Psychotherapy Change? British Journal of Psychotherapy, 26 (4), 486-501.

Ciclitira, K. et Foster, N. (2012). Attention to Culture and Diversity in Psychoanalytic Trainings. British Journal of Psychotherapy, 28 (3), 353-373.

Davids, F. (2003). The internal racist. Bulletin of the British Psychoanalytical Society 39 (4), $1-15$. 
DiAngelo, R. (2011). White fragility. The International Journal of Critical Pedagogy, 3 (3). Repéré à http://libjournal.uncg.edu/ijcp/article/view/249

Delanoë, D. et Moro, M. R. (2016). Les rapports sociaux dans le transfert culturel: Essai de problématisation. L'Autre, 17 (2), 203-211.

Fassin, D., Fassin, E. et Beaud, S. (dir.) (2009). De la question sociale à la question raciale: représenter la société française. Paris: Découverte.

Fermi, P. (1998). Le contre-transfert culturel? Repéré à http://geza.roheim.pagesperso-orange. $\mathrm{fr} / \mathrm{html} /$ contrans.htm

Flahault, F. (1978). La parole intermédiaire. Paris: Seuil

Freud, S. (1919). L'inquiétante étrangeté. Repéré à http://classiques.uqac.ca/classiques/freud_ sigmund/essais_psychanalyse_appliquee/10_inquietante_etrangete/inquietante_etrangete.html

Huqueleux, V. (2016). Du contre-transfert aux contours de l'empathie: nécessité d'une éthique clinique. L'Autre, 17 (2), 182-191.

Kaës, R. (1998). Introduction. Une différence de troisième type. Dans R. Kaës (2012), Différence culturelle et souffrance de l'identité (p. 1-19). Paris: Dunod.

Kristeva, J. (1988). Étrangers à nous-mêmes. Paris: Fayard.

Krymko-Bleton, I. (2014a). Recherche psychanalytique à l'université. Recherches qualitatives, 16 (numéro hors-série), 52-60.

Krymko-Bleton, I. (2 014b). Rencontre et discours de la méthode. Filigrane, 23 (2), 109-124.

Krymko-Bleton, I. (2016). Entre la psychanalyse et la linguistique: une démarche de recherche au sein d'un département de psychologie. Recherches qualitatives, Hors série, 20, 487-499. Repéré à http://www.recherche-qualitative.qc.ca/documents/files/revue/ hors_serie/HS-20/rq-hs-20-krymko-bleton.pdf

Menès, M. (2004). L'inquiétante étrangeté. La lettre de l'enfance et de l'adolescence, 56 (2), 21-24.

Mestre, C. et Moro, M. R. (2012). L'intime et le politique: Projet pour une ethnopsychanalyse critique. L'Autre, 13 (3), 263-272.

Moro, M. R. (2002). Le racisme: une question clinique transgressive. L'Autre, 3 (2), 345-349.

Neville, H. A., Awad, G. H., Brooks, J. E., Flores, M. P. et Bluemel, J. (2013). Color-blind racial ideology: Theory, training, and measurement implications in psychology. American Psychologist, 68 (6), 455-466.

Paillé, P. et Mucchielli, A. (2003). L'analyse qualitative en sciences humaines et sociales ( $3^{e}$ éd.). Paris: Armand Colin.

Rouchon, J.-F. (2007). La notion de contre-transfert cultuel: enjeux théoriques, cliniques et thérapeutiques. (Thèse de doctorat). Repéré à www.clinique-transculturelle.org/pdf/ these_jfr.pdf.

Rouchon, J.-F., Reyre, A., Taïeb, O. et Moro, M.-R. (2010). L'utilisation de la notion de contretransfert culturel en clinique. L'Autre, 10 (1), 80-89.

Rousseau, C. (2008). Entrevue accordée à Radio-Canada. Repéré à http://beta.radio-canada. $\mathrm{ca} /$ nouvelle/402016/accommodements-responsables

Thompson, C. E., Worthington, R. et Atkinson, D. R. (1994). Counselor content orientation, counselor race, and black women's cultural mistrust and self-disclosures. Journal of Counseling Psychology, 41 (2), 155-161.

Tissières, N. et Krymko-Bleton, I. (2018). Être psychologue et immigrant: les enjeux de la clinique. L'Autre, 19 (1), 11-20. 